\title{
CHARACTERISTICS OF AN ELECTRON-BEAM ROCKET PELLET ACCELERATOR*
}

\author{
C. C. Tsai, C. A. Foster, S. L. Milora, and D. E. Schechter \\ Oak Ridge National Laboratory \\ P.O. Box 2009 \\ Oak Ridge, TN 37831-8071
}

A proof-of-principle (POP) electron-beam pellet accelerator has been developed and used for accelerating hydrogen and deuterium pellets. An intact hydrogen pellet was accelerated to a speed of $460 \mathrm{~m} / \mathrm{s}$ by an electron beam of $13.5 \mathrm{keV}$, $0.3 \mathrm{~A}$, and $2 \mathrm{~ms}$. The maximum speed is limited by the acceleration path length $(0.4 \mathrm{~m})$ and pellet integrity. Experimental data have been collected for several hundred hydrogen pellets, which were accelerated by electron beams with parameters of voltage up to $16 \mathrm{kV}$, current up to $0.4 \mathrm{~A}$, and pulse length up to $10 \mathrm{~ms}$. Preliminary results reveal that the measured bum velocity increases roughly with the square of the beam voltage, as the theoretical model predicts. The final pellet velocity is proportional to the exhaust velocity, which increases with the beam power. To reach the high exhaust velocity needed for accelerating pellets to $>1000 \mathrm{~m} / \mathrm{s}$, a new electron gun, with its cathode indirectly heated by a graphite heater and an electron beam, is being developed to increase beam current and power. A rocket casing or shell around the pellet has been designed and developed to increase pellet strength and improve the electron-rocket coupling efficiency. We present the characteristics of this pellet accelerator, including new improvements.

\section{Introduction}

Injection of frozen pellets of hydrogen or deuterium that are up to $6 \mathrm{~mm}$ in diameter and $6 \mathrm{~mm}$ long at speeds up to a few kilometers per second has become the leading technology for fueling fusion plasmas with neutral particles [1]. Such plasma fueling can improve plasma energy confinement via modifications of plasma density and/or temperature profiles. The development at Oak Ridge National Laboratory (ORNL) of advanced pellet injectors for fueling fusion reactors, such as ITER, is discussed in Refs. [1-3]. At present, high-speed pellets are accelerated by pneumatic and/or centrifuge pellet accelerators. Many acceleration techniques (the electrothermal gun, rail gun, two-stage pneumatic gun, etc.) have been proposed and developed for accelerating pellets to high speeds [1-8]. Here, we pursue an alternative method that uses intense electron beams to accelerate pellets by means of the rocket effect $[2,9,10]$. We briefly describe acceleration theory, the test facility, and experimental results. We then discuss technical opportunities and conceptual designs of high-speed pellet accelerators.

\section{Acceleration Theory}

The basic concept is to use an intense electron beam to evaporate a hydrogen propellant "ice stick" and heat the exhaust gas to high velocity, and subsequently to accomplish rocket-like acceleration of the deuterium and/or tritium pellet payload to high speeds. Such a device is called an electronbeam (e-beam) rocket pellet accelerator. The main objective of this pellet accelerator program is to accelerate deuterium $\left(D_{2}\right)$ and/or tritium $\left(T_{2}\right)$ pellets to speeds $>10 \mathrm{~km} / \mathrm{s}$ for fueling fusion reactors such as ITER. An initial evaluation [9] indicates that a device with a 20-m-long acceleration path may be able to accelerate $D_{2}$ or $T_{2}$ pellets to speeds $>10 \mathrm{~km} / \mathrm{s}$.

The evaporation phenomena of solid hydrogen pellets under e-beam rocket acceleration are envisaged to be similar to those of pellet fueling in magnetized plasmas $[11,12]$. The neutral gas shielding model is used to describe both the energy dissipation of beam electrons and the evaporation and acceleration of pellets. Theoretically, an intense beam ablates a fraction of the pellet and creates a dense gas cloud. Subsequently, the ablated gas dissipates the beam energy, limits the power flux on the pellet surfaces, and regulates the pellet evaporation rate. The deposition of the e-beam power in the ablating gas heats and raises the exit velocity of the ablated gas, which accelerates the pellet to high velocity. As described elsewhere $[2,9,10]$, the rocket equation can be used to estimate the final pellet velocity $v_{p}$ as

$$
v_{\mathrm{p}}=v_{\mathrm{e}} \ln \left(m_{0} / m_{\mathrm{p}}\right) \quad(\mathrm{m} / \mathrm{s}),
$$

where $v_{e}$ is the gas exhaust velocity, $m_{0}$ is the pellet initial mass including propellant mass and payload mass, and $m_{\mathrm{p}}$ is the pellet final mass, equivalent to payload mass if the propellant burns up. If the propellant and payload pellets, with an initial length of $L_{0}$, are assumed to be made of a single gas and evaporation is uniform over the pellet's rear surface at a constant burn velocity $v_{\mathrm{b}}$, the final pellet velocity $v_{\mathrm{p}}$, Eq. (1), can be rewritten as

$$
v_{\mathrm{p}}=v_{\mathrm{e}} \ln \left[L_{0} /\left(L_{0}-v_{\mathrm{b}} t\right)\right] \quad(\mathrm{m} / \mathrm{s}) .
$$

Under intense ablation of electron beams with voltage $E$ (in volts) and current $I$ (in amperes) with negligible thermal evaporation energy, the neutral gas shielding model is used to derive equations for the burn velocity $v_{\mathrm{b}}$ and the exhaust velocity $v_{e}$, \footnotetext{
Research sponsored by the Office of Fusion Energy, U.S. Department of Energy, under contract DE-AC05-840R21400 with
Martin Marietta Energy Systems. Inc. 


\section{DISCLAIMER}

This report was prepared as an account of work sponsored by an agency of the United States Government. Neither the United States Government nor any agency thereof, nor any of their employees, makes any warranty, express or implied, or assumes any legal liability or responsibility for the accuracy, completeness, or usefulness of any information, apparatus, prctuct, or process disclosed, or reoresents that its use would not infringe privately owned rights. Reference herein to any specific commercial product, process, or service by trade name, trademark, manufacturer, or otherwise does not necessarily constitute or imply its endorsement, recommendation, or favoring by the United States Government or any agency thereof. The views and opinions of authors expressed herein do not necessarily state or reflect those of the United States Government or any agency thereof. 


$$
\begin{gathered}
v_{b}=\left(2 \times 10^{15} E^{2}\right) m v_{\mathrm{s}} / \mathrm{pr}(\mathrm{m} / \mathrm{s}), \\
v_{\mathrm{e}}=\left\{f 1 /\left[\pi r m v_{\mathrm{s}}\left(1 \times 10^{15} E\right)\right]\right\}^{1 / 2}(\mathrm{~m} / \mathrm{s}) .
\end{gathered}
$$

Here, $v_{\mathrm{s}}$ is the sound velocity of the exhaust gas, $m$ is the mass of the gas molecule, $\rho$ is the solid pellet density, $r$ is the pellet radius, and $f$ is the fraction of beam power heating the exhaust gas. The corresponding acceleration pressure $P$ on the pellet and acceleration path length $S$ are given as

$$
\begin{gathered}
P=v_{\mathrm{e}} \rho v_{\mathrm{i}}(\mathrm{Pa}), \\
S=v_{\mathrm{e}}\left(t+\left(t-L_{0} / v_{\mathrm{b}}\right) \ln \left[L_{0} /\left(L_{0}-v_{\mathrm{b}} t\right)\right]\right) \quad(\mathrm{m}) .
\end{gathered}
$$

If the pellet is made of hydrogen, these equations can be used to evaluate the perforniance of such a pellet accelerator. As shown in Fig. 1, they predict that a $3.37-\mathrm{mm}$ hydrogen pellet with an initial length of $12 \mathrm{~mm}$ can be accelerated in $6 \mathrm{~m}$ to a velocity of $10 \mathrm{~km} / \mathrm{s}$ by an electron beam of $20,000 \mathrm{~V}, 20 \mathrm{~A}$, and $1.5 \mathrm{~ms}$, assuming that $f=50 \%$ or half of the beam power is used in heating the exhaust gas to $8000 \mathrm{~m} / \mathrm{s}$.

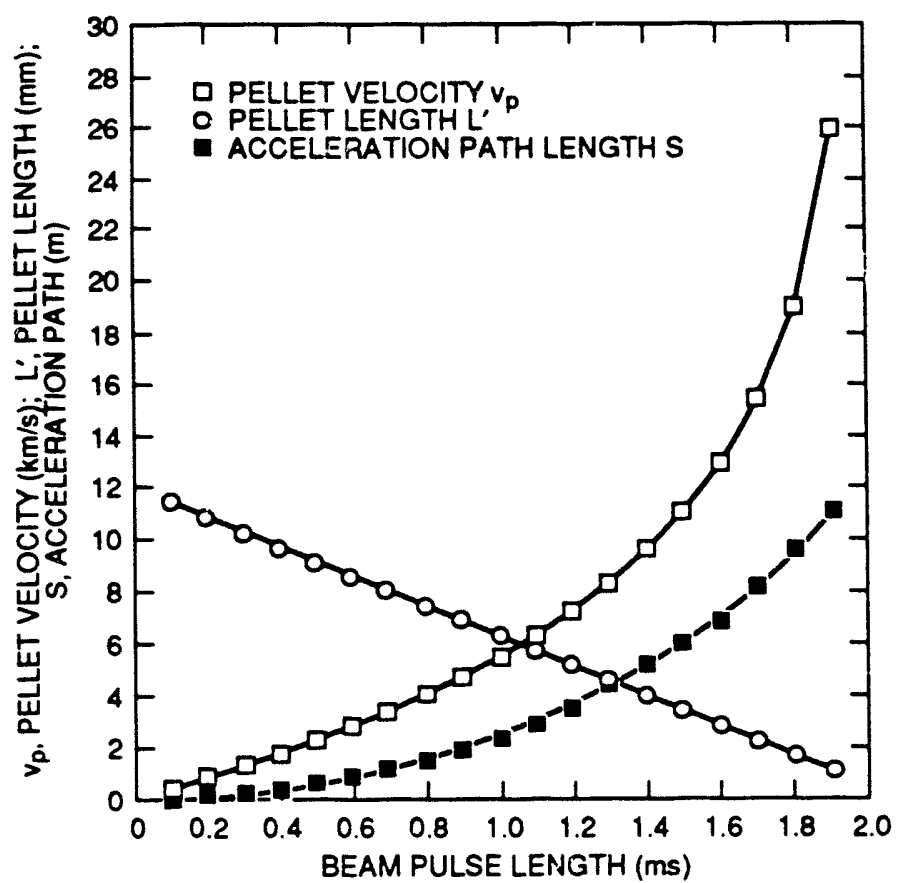

Fig. 1. Theoretically calculated pellet velocity $\nu_{p}$, pellet length $L^{\prime}$, and acceleration path length $S$ vs beam pulse length for a 12-mm hydrogen pellet with 20-kV, 10-A (or, for $f=0.5, I=20-$ A) electron beams.

\section{Test Facility}

The actual performance of an e-beam rocket pellet arielerator depends strongly on the efficiency of beam transmission, the beam ablation, the initial sound velocity of the ablated gas, the heating of the exhaust gas, etc. To demonstrate and assess the feasibility of this acceleration concept, a proofof-principle (POP) e-beam rocket pellet accelerator facility was constructed by using the mature e-beam and hydrogen pellet technology available at ORNL. Figure 2 shows the experimental arrangement of the POP device, its supporting vacuum system, and necessary diagnostic system. The device comprises a pellet maker, an electron gun, and a pellet accelerator with necessary guide rails and e-beam compression magnet. A detailed description is given in Ref. [10]. Here we briefly describe each component.

\section{A. Diagnostics}

The diagnostic system measures the parameters of pellets and electron beams. With given distances between the lighttrip detectors (labeled PD1 through PD6 in Fig. 2), a TV camera (PD7), a microwave mass detector (MD), and a shock acceleromeier (SA), the pellet speed can be estimated by measuring the time at which a pellet arrives at each sensor. The TV camera and its video system record a shadow image of the accelerated pellet. The mass of accelerated pellets can be measured by baratron capacitive gauges and the microwave mass detector next to the TV camera. The beam parameters, including current, voltage, and time, are measured by various current and voltage sensors. With Rogowski coil sensors installed at the entrance and exit ends of the guide rails, the actual beam current incident on each pellet during acceleration can be estimated. All measured data are collected and stored i y a data acquisition system and analyzed afterward.

\section{B. Automatic Pellet Maker}

For producing solid hydrogen pellets with consistent size and strength, a pellet maker has been developed to make pellets automatically, using a gas feed system under the control of a programmable logic controller (PLC). The PLC device controls pneumatic valves to feed a fixed amount of gas into a gas reservoir and to regulate gas flow into the freezing cell of the pellet maker. A cylindrical (4-mm-diam) pellet of frozen hydrogen is formed in a pipe-gun type freezing cell cooled by liquid helium to about $5 \mathrm{~K}$. The pellet length is controlled by the amount of gas feeding into the reservoir. This pellet maker makes $12-\mathrm{mm}$-long hydrogen pellets. The pellet is pushed out by a mechanical piston and launched through a baffle chamber and guide tube into the pellet accelerator with a typical initial speed between 50 and $100 \mathrm{~m} / \mathrm{s}$. The pellet maker has been equipped with a shell loading mechanism that can load a sh::i into the the freezing cell. Once the shell is loaded, the PLC device is activated to freeze hydrogen gas into the shell, thus forming a composite shell/hydrogen pellet. Pellets with shells of different materials (e.g., carbon foam, polyethylene, Teflon) have been made and launched.

\section{Electron Gun}

Many versions of the electron gun have been developed for this pellet accelerator. The electron gun uses an indirectly heated cathode with an electron emitter made of single-crystal lanthanum hexaboride, operating at $2000 \mathrm{~K}$. In studies of beam compression and transmission along the pellet acceleration column, the electre $n$ gun has been operated to form intense beams of $14 \mathrm{keV}, 11 \mathrm{~A}$, and $0.3 \mathrm{~ms}$. A solenoid magnet was used to compress the beam from $12 \mathrm{~mm}$ to $3.5 \mathrm{~mm}$. 


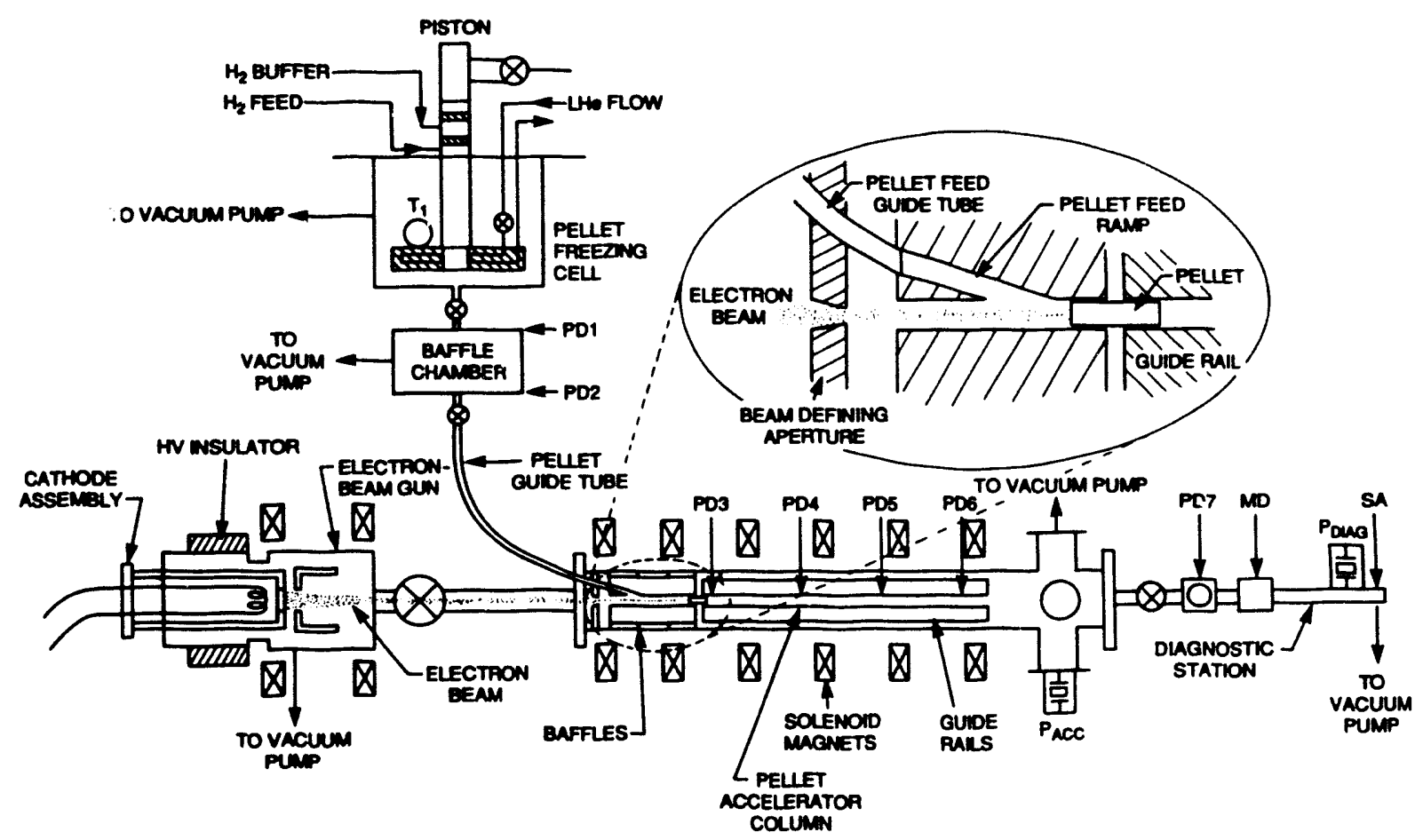

Fig. 2. Experimental setup of electron-beam rocket pellet accelerator.

A footprint of the compressed beam was made on a graphite paper sensor disk in the accelerator column [10]. The beam current density is about $10 \mathrm{~A} / \mathrm{cm}^{2}$ and potentially $>100 \mathrm{~A} / \mathrm{cm}^{2}$ at the sensor. Under high-current conditions, the electron emission cooling effect on the emitting surfaces tends to lower the beam current after a few milliseconds of beam pulse. A triode electron gun, consisting of a cathode, grid, and anode, has been developed for achieving controllable beam energy and current. Applying a negative potential of $45 \mathrm{~V}$ to the grid, the triode gun can form beams with constant but low current during the beam pulse. Electron beams with energies up to 16 $\mathrm{kV}$, pulse length up to $10 \mathrm{~ms}$, and currents up to $0.5 \mathrm{~A}$ have been transmitted through the guide rails.

\section{Pellet Accelerator}

A feed ramp loads the pellet from an off-axis trajectory in the guide tube into the acceleration column formed by three 0.6-m-long graphite guide rails (Fig. 2). The pellet can be accelerated at any location along the guide rails by the compressed e-beam. The solenoid magnet aligns, guides, and confines the compressed e-beam during pellet acceleration. The geometrical variation along the beam trajectory can substantially change local gas density and influence beam straggling and pellet acceleration. Thus, the pellet location at which the beam is applied has been optimized for pellet acceleration. In this setup, the acceleration path of the guide rails is optimized to about $0.4 \mathrm{~m}$.

\section{Results and Analysis}

Experimental data have been collected for hundreds of bare hydrogen pellets, which were accelerated by e-beams with parameters of yoltage up to $16 \mathrm{kV}$, current up to $0.4 \mathrm{~A}$, and pulse length up to $10 \mathrm{~ms}$. A typical speed increment of 300 to $500 \mathrm{~m} / \mathrm{s}$, increasing with beam power, is observed. Intact hydrogen pellets have been accelerated to speeds of 460 $\mathrm{m} / \mathrm{s}$ by an e-beam of $13.5 \mathrm{keV}, 0.3 \mathrm{~A}$, and $2 \mathrm{~ms}$. Pellets with carbon foam shells have been accelerated to speeds of about $410 \mathrm{~m} / \mathrm{s}$ with larger payload pellets. Figure 3 shows typical average pellet velocities between position monitors along the pellet path for various accelerated bare hydrogen pellets.

A preliminary analysis of accelerated bare pellets indicates that the measured bum velocity increases roughly with the square of the beam voltage, as the theoretical model predicts. This feature is apparent in Fig. 4, which shows the experimental and theoretical burn velocities of hydrogen pellets as a function of e-beam voltage. The curve for the theoretical burn velocity, from Eq. (3), is based on the assumption that the sonic velocity of the ablated gas is about $375 \mathrm{~m} / \mathrm{s}$ for a gas temperature of $24 \mathrm{~K}$.

We have attempted to analyze the exhaust velocity of the ablated gas as a function of beam power. We note that an accurate beam current measurement is essential to this analysis. However, the power of electron beams with constant current during pellet acceleration is relatively low. The thermal evaporation energy, $H_{\mathrm{v}}$, cannot be neglected in calculating the exhaust velocity. The beam power, $f I E$, equals the sum of the power for pellet evaporation, $\pi r^{2} \rho v_{\mathrm{b}} H_{\mathrm{v}}$, and the power for heating the gas, $\pi r^{2} v_{\mathrm{b} \rho} v_{\mathrm{gas}}{ }^{2} R$. Thus, the gas velocity, $v_{\mathrm{gas}}$, and the exhaust velocity, $v_{\mathrm{e}}$, can be derived as

$$
\begin{gathered}
v_{\mathrm{gas}}=\left\{f /\left[\pi \cdot r m v_{\mathrm{s}}\left(1 \times 10^{15} E\right)\right]-2 H_{\mathrm{v}}\right\}^{1 / 2}(\mathrm{~m} / \mathrm{s}) \\
v_{\mathrm{e}}=\alpha v_{\mathrm{gas}}(\mathrm{m} / \mathrm{s}),
\end{gathered}
$$




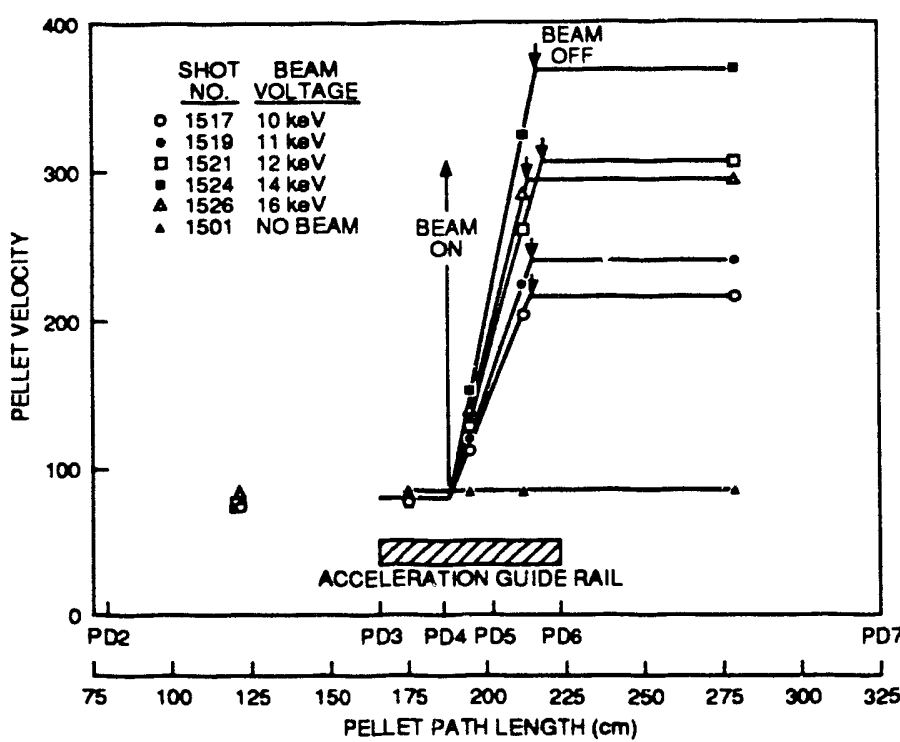

Fig. 3. The average pellet velocity between monitors ir Fig. 2. The pellet is accelerated in a 40-cm-long section identified as "acceleration guide rail." Arrows indicate beam turn-on and turn-off. Increasing the beam voltage raises the beam power and thus the pellet velocity except for the $16-\mathrm{kV}$ pulse.

where $\alpha v_{\text {gas }}$ is the average vectorial velocity of the heated exhaust gas used to accelerate the pellet. Assuming $f=1$ and $\alpha=$ 1 , the calculated pellet velocities increase with the beam current (or the beam power) as the measured velocities do. The curves in Fig. 5 indicate that the measured pellet velocities are about $40 \%$ of the maximum theoretical velocities. If this scaling feature can be maintained for higher beam currents or powers, the pellet velocity will increase to values above 1000 $\mathrm{m} / \mathrm{s}$. In fact, hydrogen pellets could theoretically be accelerated to about $2200 \mathrm{~m} / \mathrm{s}$ without the limitation of the acceleration path length and to about $1300 \mathrm{~m} / \mathrm{s}$ with a $0.5-\mathrm{m}$ acceieration path by $14-\mathrm{kV}$ beams of $1 \mathrm{~A}$ and $1.3 \mathrm{~ms}$. Thus, higher exhaust and pellet velocities would be obtained with higher beam currents and longer acceleration lengths.

\section{Discussion}

Based on these significant results, this acceleration technique appears able to achieve a performance level competitive with other state-of-the-art acceleration techniques. To develop a high-speed $(>1-\mathrm{km} / \mathrm{s})$ pellet accelerator, the essential technical approaches are

1. increasing the beam power or beam current to increase exhaust velocity,

2. using a shelled pellet to enhance acceleration and maintain pellet integrity during intense beam acceleration, and

3. extending the acceleration path length to a few meters (compared with the present $0.4-\mathrm{m}$ accelerator).

Steps 1 and 2 have been pursued and implemented in the present experimental setup; plans are to carry out step 3 in an upgraded POP device. The electron gun has been modified and upgraded to deliver higher beam powers or currents. The development effort on the t-beam system is devoted to

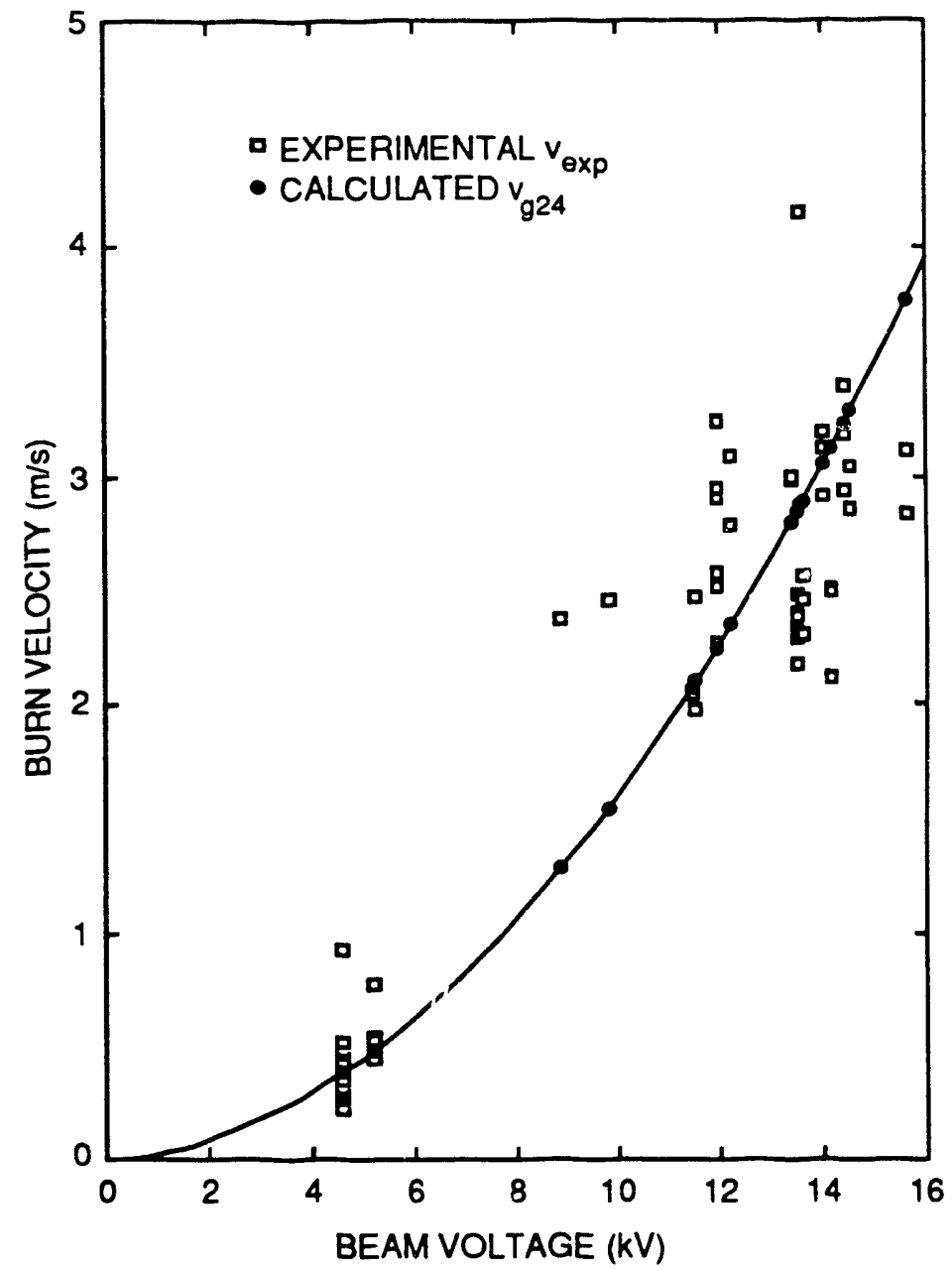

Fig. 4. Measured burn velocity of pellets as a function of beam voltage $E$ compared with the neutral shield model, which predicts an $E^{2}$ dependence. The "burn velocity" is the rate at which the solid hydrogen pellet ablates under the influence of the electron beam. The nomenclature is taken from that used for chemical solid fuel rockets, which actually do burn.

improving beam transmission and beam optics ânu minimizing emission cooling. To compensate for the emission cooling, the electron gun has been modified with another e-beam heater in addition to the original cathode heater. This new cathode offers opportunities to develop alternative electron guns, which could form off-axis high-current e-beams for future multi-stage pellet accelerators, as discussed below. An electron gun with a new extractor and accelerator has been designed for improved optics and higher current. The solenoid magnet and the mechanical genmetry along the beam path are also being modified to improve beam transmission.

The goal of achieving high pellet velocity by increasing beam power may be hindered by insufficient pellet strength. To enhance pellet strength, we modified the pellet maker to form hydrogen pellets in lightweight casings or shells made of carbon foam (CF) and polyethylene. Shelled hydrogen pellets have been successfully accelerated. The shells of CF-shelled pellets can be burned away by beam electrons. However, for the composite polyethylene/hydrogen pellets, the hydrogen 
pellet is detached prematurely. Data on accelerated CF-shelled pellets will be collected and used in studying burn velocity, acceleration pressure, and coupling efficiency of heated, exhausted gas. The merits of shelled pellets can be further enhanced if the pellet diameter is enlarged to increase beam current and to reduce the mass ratio of shell to propellant pellet. Future efforts will be devoted to increasing $v_{\mathrm{p}}$.

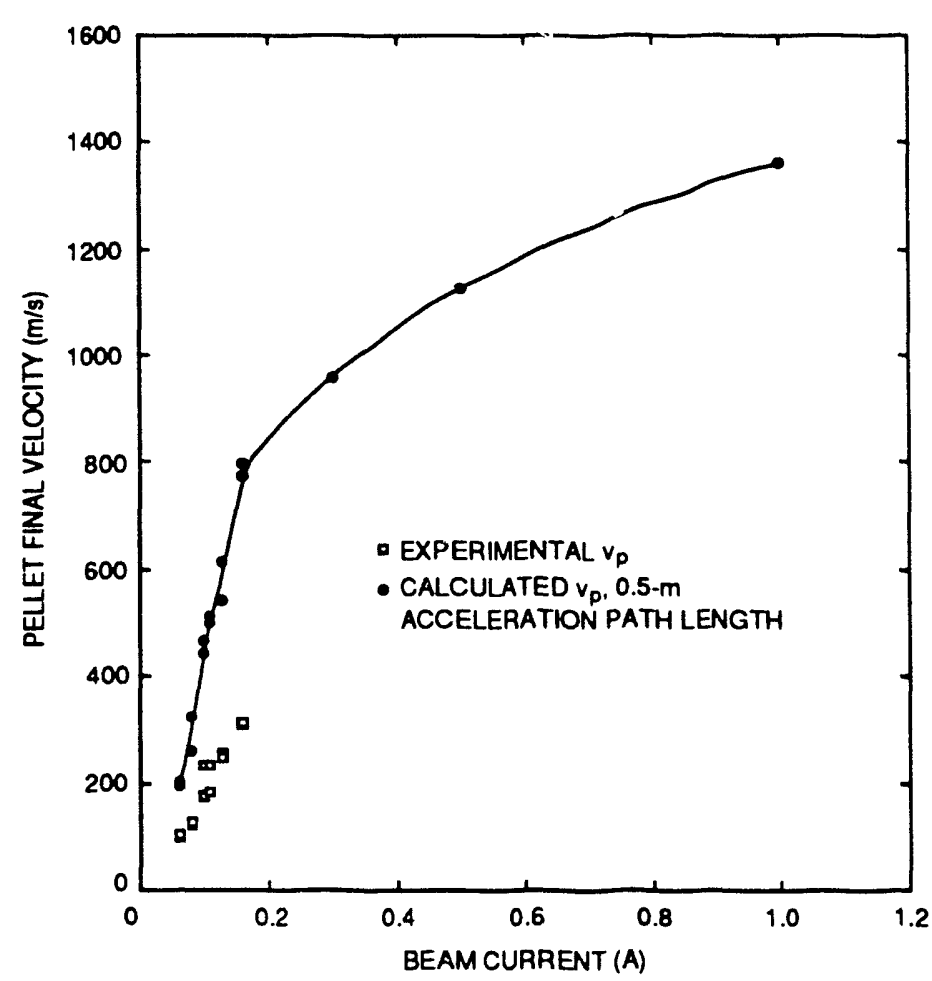

Fig. 5. Final velocity of the pellet as a function of the electron beam current. Calculated values are from Eqs. (2), (3), (7), and (8), with the parameters $f=1$ and $\alpha=1$, for various beam voltages and pulse lengths.

Figure 1 shows that long acceleration paths $(\sim 1 \mathrm{~m})$ are needed to achieve velocities of $>1 \mathrm{~km} / \mathrm{s}$. Therefore, step 3 , increasing the acceleration length by several meters, is essential to achieving ultrahigh speeds of $5 \mathrm{~km} / \mathrm{s}$. In 1992, we will attempt to accelerate pellets to $>1 \mathrm{~km} / \mathrm{s}$ by extending the acceleration path length of the apparatus to about $1 \mathrm{~m}$ (compared with the present $0.4-\mathrm{m}$ acceleration path). This work will confirm the merits of pellet acceleration theory. Our conceptual ultrahigh-speed pellet accelerator is a device with multistage acceleration capability, as discussed in Ref. [10] and shown in Fig. 6. We envision that this pellet accelerator could be formed by many short modular accelerators, each $\approx 1 \mathrm{~m}$ long. In one modular acceleration stage, an upgraded POP pellet accelerator can use an off-axis hollow e-beam to accelerate long, shelled pellets to be launched axially. After demonstrating the reliability and feasibility of the pellet accelerator, we can extend the acceleration path simply by adding more modules.
We view these steps as a necessary scaling effort for extrapolating the performance of the e-beam acceleration concept and developing a pellet injector with a long acceleration path $(\sim 20 \mathrm{~m})$ to fulfill the ITER high-velocity $(>10-\mathrm{km} / \mathrm{s})$ objective.

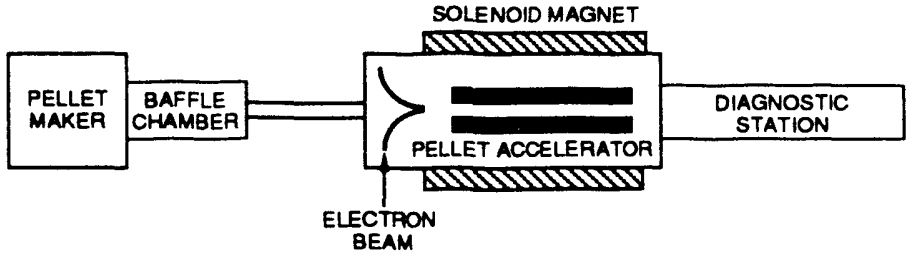

Fig. 6. A conceptual ultrahigh-speed pellet accelerator with a single acceleration module.

\section{References}

[1] S. L. Milora, "Review of Pellet Fueling," J. Fusion Energy, vol. 1, p. 15, 1981; "Review of Hydrogen Pellet Injection Technology for Plasma Fueling Applications," J. Vac. Sci. Technol. A, vol. 7, pp. 925-937, 1989; these proceedings.

[2] C. A. Foster, C. C. Tsai, D. E. Schechter, et al., "The ORNLTore Supra Pellet Injector and the e-Beam Rocket Pellet Accelerator," in Pellet Injection and Toroidal Confinement: Proceedings of the IAEA Technical Committee Meeting, Gut Ising, 24-26 October 1988, 1989, p. 275.

[3] S. L. Milora, S. K. Combs, C. A. Foster, et al., "Pneumatic Pellet Injector Research at ORNL," ibid., p. 709.

[4] C. A. Foster, R. J. Colchin, S. L. Milora, K. Kim, and R. J. Tumbull, Nucl. Fusion, vol. 17, p. 1067, 1977.

[5] S. K. Combs, S. L. Milora, C. R. Foust, C. A. Foster, and D. D. Schuresko, Rev. Sci. Instrum., vol. 56, p. 1173, 1985.

[6] D. D. Schuresko, S. L. Milora, S. K. Combs, C. R. Foust, B. E. Argo, G. C. Barber, C. A. Foster, and N. S. Ponte, J. Vac. Sci. Technol. A, vol. 5, p. 2194, 1987.

[7] P. W. Fisher, M. L. Bauer, L. R. Baylor, et al., "Tritium Pellet Injector Results," J. Vac. Sci. Technol. A, vol. 7. pp. 938-943, 1989.

[8] S. K. Combs, C. R. Foush, M. J. Gouge, and S. L. Milora, "Acceleration of Small, Light Projectiles (Including Hydrogen Isotopes) to High Speeds Using a Two Stage Light Gas Gun," $J$. Vac. Sci. Technol. A, vol. 8, p. 1814, 1990.

[9] C. A. Foster and M. M. Menon, "Electron Beam Rocket Pellet Accelerator," presented at the International Pellet Fueling Workshop, La Jolla, Califomia, October 30-November 3, 1985.

[10] C. C. Tsai, C. A. Foster, and D. E. Schechter, "Characteristics of an Electron-Beam Rocket Pellet Accelerator," in Proceedings of the IEEE 13th Symposium on Fusion Engineering, 1989, pp. 1230-1235.

[11] S. L. Milora and C. A. Foster, Rev. Sci. Instrum., vol. 50, p. 482, 1979.

[12] P. B. Parks, R. J. Tumbull, and C. A. Foster, Nucl. Fusion, vol. 17, p. 539, 1977. 

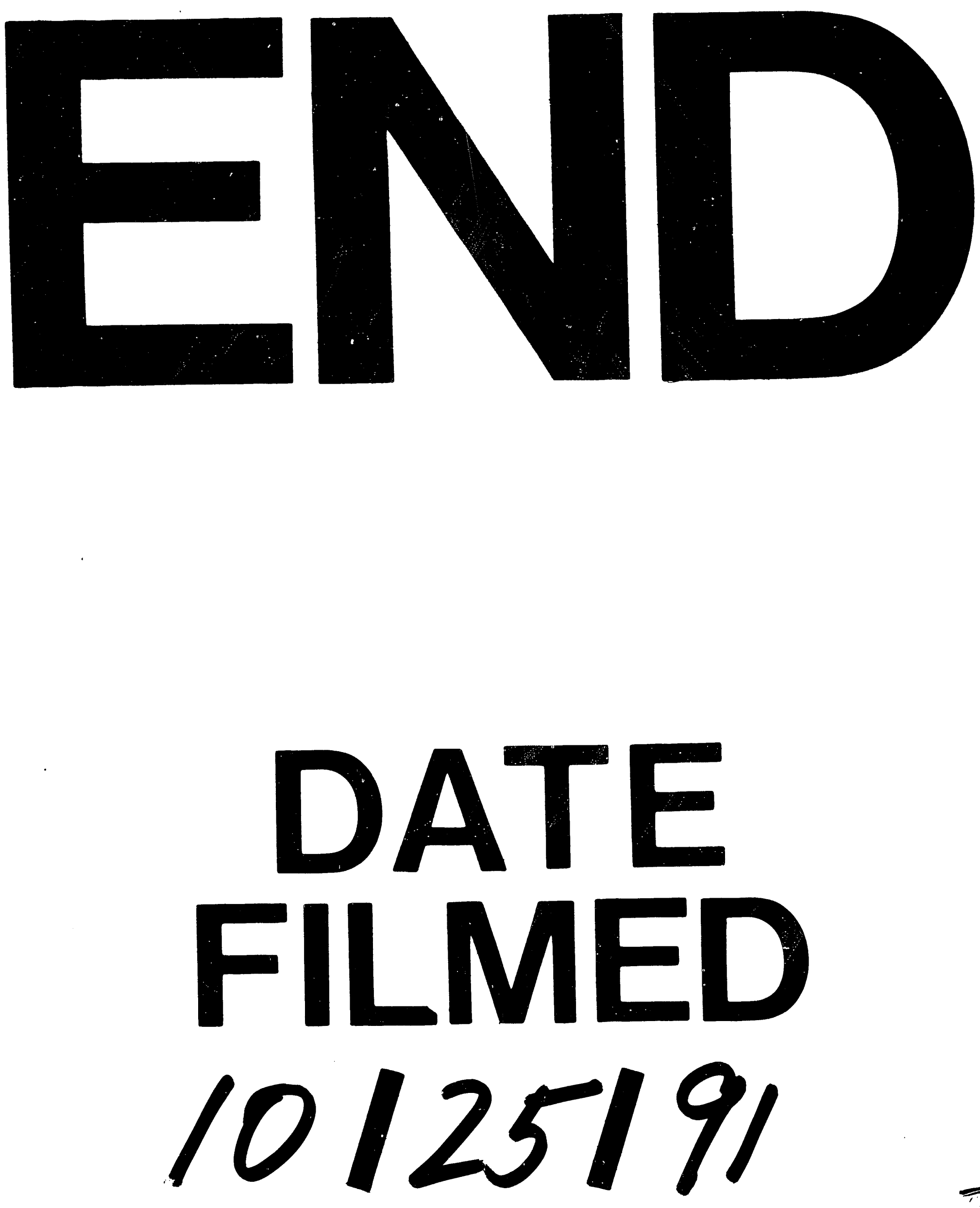
